\title{
Curvilinear Structure Enhancement with the Polygonal Path Image - Application to Guide-Wire Segmentation in X-Ray Fluoroscopy
}

\author{
Vincent Bismuth $^{1,2}$, Régis Vaillant ${ }^{2}$, Hugues Talbot $^{1}$, and Laurent Najman ${ }^{1}$ \\ 1 Université Paris est, laboratoire d'informatique Gaspard-Monge, équipe A3SI, \\ ESIEE, 77454 Marne-la-Vallée Cedex 2, France \\ vincent.bismuth@med.ge.com \\ 2 General Electric Healthcare, 283 rue de la minière, 78533 Buc, France
}

\begin{abstract}
Curvilinear structures are common in medical imaging, which typically require dedicated processing techniques. We present a new structure to process these, that we call the polygonal path image, denoted $\mathfrak{P}$. We derive from $\mathfrak{P}$ some curvilinear structure enhancement and analysis algorithms. We show that $\mathfrak{P}$ has some interesting properties: it generalizes several concepts found in other methods; it makes it possible to control the smoothness and length of the structures under study; and it can be computed efficiently. We estimate quantitatively its performance in the context of interventional cardiology for the detection of guide-wires in Xray images. We show that $\mathfrak{P}$ is particularly well suited for this task where it appears to outperform previous state of the art techniques.
\end{abstract}

Keywords: curvilinear structures, image segmentation, shortest path.

\section{Introduction}

Curvilinear structures appear naturally in the human body and thus in medical images. Their segmentation is a specific but extensively studied topic that covers a wide variety of naturally elongated biological structures and medical tools: vascular and cerebral structures and interventional tools like catheters, guide-wires, etc. In this paper, we propose a locally shortest path technique for the processing of curvilinear structures. The proposed technique associates a generalization of several state of the art techniques, an intuitive parameterization, and an efficient computational scheme. We illustrate its performance for the difficult task of guide-wire segmentation in X-ray fluoroscopy. We propose quantified results on clinical data and comparison with other state of the art techniques.

\section{Background}

\subsection{Guide-Wire Detection in X-Ray Fluoroscopy}

Interventional radiology/cardiology therapies imply inserting guide-wires into the vascular system of patients under the monitoring of X-ray video, called fluoroscopy. Such procedures are minimally invasive and have been used increasingly 
often in recent years, in areas ranging from coronary angioplasty to tumor embolization. Over the past years, guide-wire detection in fluoroscopic images has gained interest and maturity among the image processing community \begin{tabular}{ll|l|l|l}
1 & 2 & 3 & 4
\end{tabular} . A large number of applications rely upon its characteristics, such as visualization enhancement, 3D guide-wire reconstruction and respiratory motion tracking. In X-ray images, guide-wires appear as thin, dark curves (see Fig. 11(a)). The challenge in their detection arises from their low contrast to noise ratio and the superposition with disturbing clutter and anatomical structures.

\subsection{State of the Art}

Curvilinear structure segmentation techniques and particularly guide-wire detection in X-ray fluoroscopy is often presented as a 3-step pipeline: (1) The local building of a feature map representing the probability of presence of an elongated structure at each pixel [2 $345[6]$. Its computation often involves considering the neighborhood of each pixel, on which structures are assumed to be straight. (2) A semi-local feature map reinforcement, typically enhancing responses aligned along a pattern - e.g. $2^{\text {nd }}$ order, circle or parabola 1, or tensor voting and coherence enhancing diffusion. (3) A global structure segmentation. At this stage, simple operators like thresholding are generally not sufficient, and a higher level process is invoked to segment the feature map. It may involve grouping [34 or tracking [6].

However some techniques, like locally shortest paths [7] and geodesic path voting [8] are exceptions to this framework. They discard complex curve models (linear, $2^{\text {nd }}$ order etc.) instead relying on the concept of path. Locally shortest paths associate a locally optimal path to every pixel and use the cost of such paths for segmentation. Geodesic path voting relies on a starting point on the structure to segment from which a set of shortest paths are computed to a large number of automatically determined endpoints in the image. The geodesic density (number of paths passing through a pixel) is used to enhance lines.

\section{Method}

\subsection{The Polygonal Path Image}

Presentation: As noted above, most line enhancement techniques select at each pixel a curve that best fits locally following a model: line segment, $2^{\text {nd }}$ order model or "arbitrary" smooth curves. We propose a single tunable model that generalizes these steps. Our local curve model is a path, that is characterized by length, and smoothness. Our aim is still to select at each pixel a best-fitting curve of given smoothness and length. However, instead of assigning to each pixel a measure associated to a local curve fitting, we propose to use the whole set of locally fitted curves over the image to derive more comprehensive line enhancement techniques.

The smoothness of the paths is controlled by considering regular polygonal paths defined by two parameters: a total arclength $L$ and a length $l$ of every 
line segment. These two parameters have a simple intuitive meaning and are set according to the properties of the structure to enhance. The polygonal constraint is a generalization of the classical local path approach [7] (for $l=1$ ) enabling the encoding of the a-priori tortuosity of the structures to detect. From a higher level perspective, $L$ is equivalent to the steps of the previous section. Indeed a single segment in the polygon is equivalent to the local step (i.e. segment matching). Considering a few segments is like semi-local processing, reinforcing the aligned responses of the local step. Finally a large number of segments takes into account long paths as in the global step.

Definition of $\mathfrak{P}$ : We consider a potential image $I$ of strictly positive values, with structures of interest exhibiting lower values. For each pixel $p \in I, \Phi_{p}$ is a set of admissible paths. We assign to each such path $\mathcal{P} \in \Phi_{p}$ a $\operatorname{cost} J(\mathcal{P})$ that is the sum of the values of $I$ along $\mathcal{P}$ (Eq. 1). The path yielding the lowest cost in $\Phi_{p}$ is denoted the locally optimal path $\mathcal{P}_{p}^{*}$ at pixel $p($ Eq. 2$)$. The set of admissible paths is defined by some constraints: Paths shall be regular polygonal lines of given length $L$, and shall have controlled curvature: at each pixel the path shall be included inside a cone of given orientation and aperture [7]. These constraints enforce locality, the smoothness that is often expected in medical images, and basically defines a search range around each pixel. We call the "polygonal path image" (noted $\mathfrak{P}$ ) the structure that contains a path and its cost for each pixel.

$$
J(\mathcal{P})=\sum_{p \in I} I(p) . \mathbf{1}_{\mathcal{P}}(p) \quad(1) \quad \mathcal{P}_{p}^{*}=\underset{\mathcal{P} \in \Phi_{p}^{L}}{\operatorname{argmin}} J(\mathcal{P})
$$

Complexity: The computation of $\mathfrak{P}$ with a brute force approach has exponential complexity with regard to $L: O\left(L k^{L}\right)$. Computing all the locally optimal paths in the image for $n$ polygonal segments before considering the optimal paths of $n+1$ segments, allows us to achieve linear complexity $O(L)$ by adapting the original algorithm of Vincent [7]. This makes it possible to consider $\mathfrak{P}$ with long paths (e.g. one hundred pixels). For example, it takes approximately 10 s to process a $512^{2}$ image with such long paths. In terms of memory, we require the initial image times the number of polygon segments to store the paths.

\subsection{Structure of the Path Image}

Some paths originating from random locations on a clinical image are shown on Fig. 1 (b). We note that paths tend to converge into bundles around the main linear structures, where the cost in the potential image is lower. Those originating outside of the linear structures take the shortest possible way to reach them. Those that start directly on linear structures stay on them until the end or until paths constraints are exceeded. High path density is thus characteristic of the linear structures. Also, the set of paths intersecting at a pixel convey some local geometry information on the linear structures. Fig. 1 (c) illustrates paths 


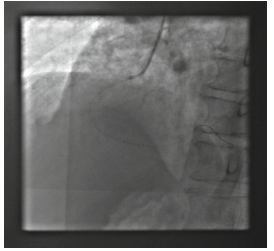

(a)

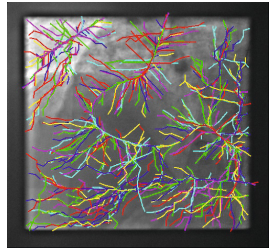

(b)

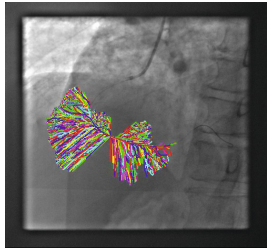

(c)

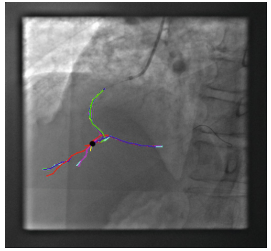

(d)

Fig. 1. (a) X-ray fluoroscopy image from an angioplasty exam illustrating a guide-wire, with a long smooth curve appearance and low contrast to noise ratio. (b): 500 locally optimal paths originating from random locations. Observe their tendency to converge to the linear structures of the image and especially to the guide-wire. (c,d) The set of paths intersecting at one given point (belonging to the guide-wire, in (c), and to the background in $(\mathrm{d})$, in this case the point is indicated by the dark spot).

intersecting at a point on the guide-wire. We observe (i) a high number of paths crossing at this point - nearly 11000 in this case (ii) that they are aligned with the guide-wire in a small neighborhood around the considered pixel.

\subsection{Image Processing with $\mathfrak{P}$}

Path Voting: We observed that paths form bundles around the main linear structures. Therefore we propose that each minimal path vote for all the pixels it passes through, to reinforce path overlapping. This approach is similar to that of Carlotto [9] and to geodesic voting [8], which also perform voting on sets of paths. Several voting schemes are possible: e.g. each vote can count the same, which is equivalent to counting the number of paths intersecting at one pixel (we note the resulting image $\vartheta(\mathfrak{P})$ - Eq. 3), or each vote can be weighted (with a value denoted $\vartheta_{w}(\mathfrak{P})$ Eq.4). We can also restrict voting to the paths that meet some a priori criteria, e.g. preventing very tortuous paths from voting. Such paths occur in parts of the image were there is little relevant linear structure and close to high contrast objects. To this end, we define a tortuosity metric $\tau$ to penalize abrupt changes in direction, i.e. 0 for a path with a change of $\pi / 2 \mathrm{rad}$ and 1 for a perfectly straight path. $\tau(\mathcal{P})$ is given by Eq. [5 (where $\mathbf{V}(k)$ is the vector formed by two consecutive vertexes of $\mathcal{P}$ ). The operation of voting with a path smoothness constraint is denoted $\vartheta_{\tau_{\min }}(\mathfrak{P})$. Its result is illustrated in Fig. 2(b).

$$
\begin{gathered}
\vartheta(\mathfrak{P})=\sum_{p \in I} \mathbf{1}_{\mathcal{P}_{p}} \\
\tau(\mathcal{P})=\frac{1}{l^{2(n-2)}} \prod_{k=1}^{n-2} \mathbf{V}(k) \cdot \mathbf{V}(k+1)
\end{gathered}
$$

$$
\begin{aligned}
\vartheta_{w}(\mathfrak{P}) & =-\sum_{p \in I} w(p) \mathbf{1}_{\mathcal{P}_{p}} \\
\vartheta_{\tau_{\text {min }}}(\mathfrak{P}) & =\sum_{\substack{p \in I \\
\tau\left(\mathcal{P}_{p}\right)>\tau_{\text {min }}}} \mathbf{1}_{\mathcal{P}_{p}}
\end{aligned}
$$

Pruning in $\mathfrak{P}$ : Another way exploiting the structure of the path image is to prune paths so as to select only a small set of relevant and non-redundant locally 


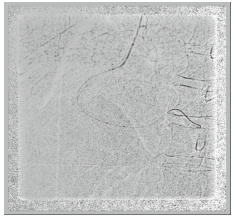

(a)

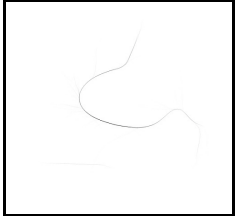

(b)

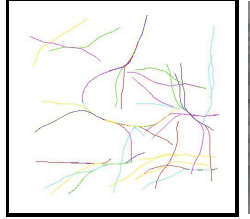

(c)

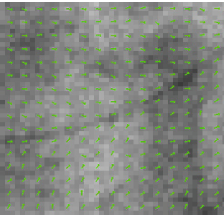

(d)

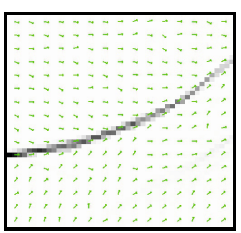

(e)

Fig. 2. (a) Dark top-hat of Fig. 1 (a), used as potential image. (b) Result of $\vartheta_{\tau_{\min }=0.6}$. Despite a low CNR the guide-wire is significantly enhanced. (c) Path pruning with $x=50 \%$ and $\tau_{\text {min }}=0.75$. Observe how the conjunction of pruning and constrained path smoothness help segment the guide-wire. (d) Close-up on a guide-wire segment in Fig. 1 (a) overlaid with the directions extracted from $\mathfrak{P}$. They indicate the direction of the closest curvilinear structure. On the structure itself the direction of the arrow is not relevant, but the orientation is accurate. (e) Same direction field overlaid on $\vartheta_{\tau_{\min }}$.

optimal paths. To do so, we define a neighborhood relationship between paths based on the partial Hausdorff distance. Then, we select the path of minimal cost in $\mathfrak{P}$ and prune all the neighboring paths. We iterate this process in a greedy fashion, selecting the path of minimal cost again and pruning its neighbors, until there is no more path in $\mathfrak{P}$ or some stopping criterion is reached. The remaining set of paths (typically less than 100) describes the linear features in the image (see Fig. 2 (c)).

Estimating Local Orientation: We have illustrated in Fig. 11 that, when they reach a linear structure, paths tend to follow and align with it. Therefore the set of paths intersecting at a pixel on a curvilinear structure can be used to derive its orientation (an angle in $[0, \pi[$ ). Outside of the curvilinear structures the paths follow the shortest path leading to a linear structure. Their direction thus indicates the shortest path to line-like structures. To estimate it, let us consider at a given pixel $p$, the set of the paths $\left\{\mathcal{P}_{i}\right\}$ intersecting at $p$. For each of these paths, we compute the tangent unit vector at point $p:\left\{\mathbf{t}_{i}\right\}$. Then we find the unit vector $\mathbf{v}_{p}$ yielding the maximal sum of scalar products with the $\left\{\mathbf{t}_{i}\right\}$. This method is illustrated in Fig. 2 (d, e).

\subsection{Evaluating and Comparing Line Enhancement Methods}

To quantify the performance of our line enhancement techniques, we use ROC analysis to characterize their ability to assign larger values to the pixels of the structure of interest rather than to the background pixels. The Area Under the ROC Curve (AUC) is an estimate of the probability that a classifier will rank a randomly chosen pixel of the structure higher than a randomly chosen pixel of the background. When the number of background pixels far exceeds the number of pixels of the structure of interest, the computation of the AUC can be restricted to the low false positive rate range, in our case $[0,5 \%]$. We normalize the AUC by the range, i.e. $5 \%$ here. In this context a random guess 
would yield a performance of only $2.5 \%$ and a perfect detector $100 \%$. This metric is denoted the "partial AUC". In order to illustrate further the performance of the method we compute the false positive rate for a fixed true positive rate of $90 \%$. Obviously, the better the method, the lower this metric. To compute these metrics, a trained operator manually defined ground truth $\mathcal{T}$ for each image, representing the centerline of the structure. We denote by $\mathcal{D}$ the set of points detected in an image for a given threshold. In order to take into account the real width of the curvilinear structure, we define true positives as the pixels of $\mathcal{T}$ that lie closer to $\mathcal{D}$ than a pre-defined distance $d$ (typically the radius of the guide-wire). False positives are defined as the detected pixels that lie further from $\mathcal{T}$ than $d$. To make our results unbiased w.r.t. the line-width parameter of the line enhancement techniques, we skeletonize the detected pixels before computing the detection rates. Finally, in order not to corrupt our assessment of line enhancement techniques with the presence of other interventional tools or similar curvilinear structures we also compute the metrics associated to false positives in an ROI around the marked truth.

\section{Results}

Our clinical case database consists of 12 clinical sequences of 9 images each, for a total of 108 images. These images depict angioplasty exams with the injection catheter, the guide-wire, the angioplasty balloon, the anatomical background, and occasionally, stents and sternal wires. We compared our technique to two state of the art line enhancement techniques : Frangi's Vesselness [5] and Rotated Filter Bank (RFB) [2]. They were found in several recent publications [2]10] to constitute the current state of the art for methods that compute in each pixel the probability to lie in a linear structure. Note that we are aware of dedicated methods for guide-wire segmentation that return a high level object describing the guide-wire [3]. However, these methods typically rely on low-level descriptors, and so can benefit from the type of work we present here. We hand-optimized the set of parameters for each technique independently. Regarding Vesselness, we set $\alpha$ and $\beta$ to the values proposed in Frangi's article [5]. The scale factor $\sigma$ was set to 2 pixel: the approximate guide-wire radius. For the RFB we relied on a study [10] that concluded that a length of 61 and a width of 3 pixels was optimal for this application. For $\vartheta(\mathfrak{P})$ and $\vartheta_{\tau_{\min }}(\mathfrak{P})$, we set $: l=21, L=210, \tau_{\min }=0.6$. The potential image on which the path costs are computed was obtained with a simple morphological dark top-hat.

We computed the mean $\left(\mathrm{AUC}_{\mu}\right)$ and standard deviation $\left(\mathrm{AUC}_{\sigma}\right)$ of the $\mathrm{AUC}$ over the database and the FPR for a given TPR of $90 \%$. We report them graphically in Fig. 3. We observe that the line enhancement techniques derived from $\mathfrak{P}$ performed significantly better. This is exemplified by greater AUR values and lower FPR. The local descriptors RFB and Vesselness performed similarly, within a few percents whereas $\mathfrak{P}$ achieves more than twice their performance. In order to illustrate the performance of the different techniques, we selected an optimal threshold that minimizes the sum of the missed detection rate and the 
false detection rate. We illustrate the thresholded images obtained with the four methods in Fig. 3. The outcomes are well aligned with the conclusions driven from the partial AUC: $\mathfrak{P}$ based methods perform significantly better. We observe that the results are generally speaking quite satisfactory: most of the guide-wire is segmented, and only a few false positives remain in the background. We can observe how the smoothness constraints in $\vartheta_{\tau_{\min }}(\mathfrak{P})$ removes some anatomical false positives that yield tortuous paths. However two problems remain: Over the whole database, the guide-wire tip, which is very contrasted attracts too many paths (creating false positives in its vicinity) and is too tortuous to be accurately fitted by our detector with the same setting as the guide-wire body - It must be detected separately. Secondly, some linear structures are detected in the background that are not guide-wires. Since they satisfy all the properties that we selected for guide-wires, a higher level processing, based on other criteria can handle them. For instance the presence of a tip is very characteristic of the guide-wire, as well as the motion that animates it.

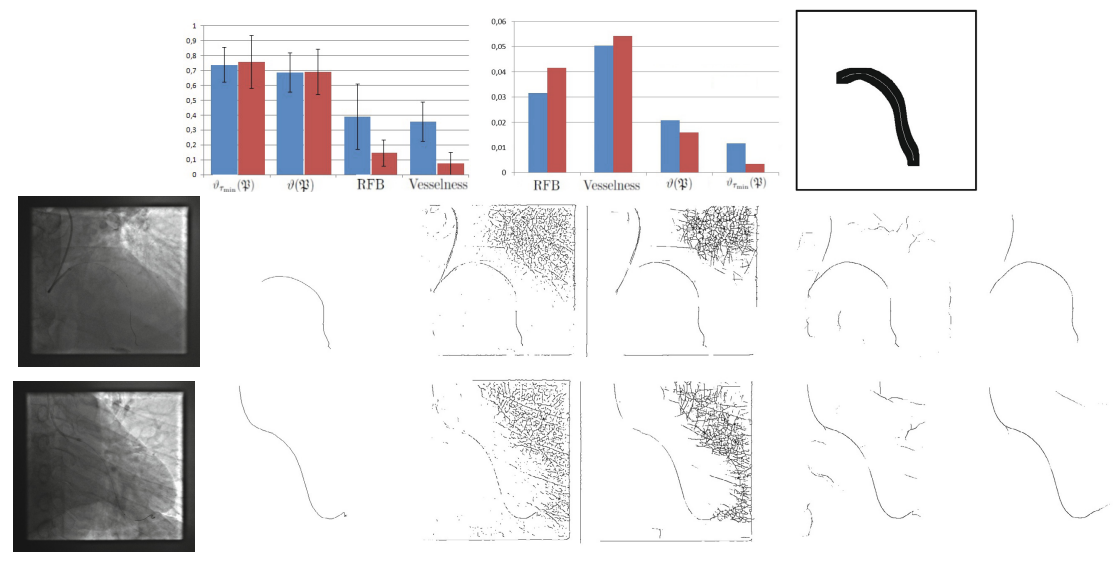

Fig. 3. Top : Performance of the four line enhancement techniques over our database. From left to right: AUC results, the height of the columns is $\mathrm{AUC}_{\mu}$ and the error bars represent mean $\mathrm{AUC}_{\mu} \pm \mathrm{AUC}_{\sigma} . \mathrm{FPR}$ for $\mathrm{TPR}=90 \%$. In both graphs the red series is computed over the whole image and the blue one only inside the ROI. The ROI used for FP is a band around the ground truth (see right image). Bottom rows: 2 result examples for optimal thresholds. From left to right : input image, ground truth, Vesselness, RFB, $\vartheta(\mathfrak{P})$ and $\vartheta_{\tau_{\min }}(\mathfrak{P})$. Observe that traditional methods enhance a large amount of non relevant structures in the background and fail to enhance some parts of the guide-wire. These problems are not present with $\vartheta_{\tau_{\min }}(\mathfrak{P})$. Note these are the exact results of the techniques, no pruning has been performed for the $\mathfrak{P}$ based methods.

\section{Conclusion and Further Work}

We have presented a new curvilinear structure processing scheme, the polygonal path image. We have demonstrated its suitability for the task of guide-wire 
detection in X-ray fluoroscopy. We showed that $\mathfrak{P}$ has several interesting properties: (i) the ability to control the smoothness and length of the structures to be analyzed, (ii) a unification of local, semi local, and global curvilinear structure analysis in a single framework and (iii) an efficient computational scheme. This structure is a rich descriptor of the curvilinear structures present in images from which we derived several tools: line enhancement, segmentation and direction field computation techniques. We have demonstrated the relevance of polygonal path voting schemes for guide-wire segmentation quantitatively in the ROC analysis formalism and compared it to state of the art techniques. Future work may include some natural extensions of the usage/construction of $\mathfrak{P}$, including enhancement and segmentation operators on the path image and the quantitative evaluation of the direction fields and of path pruning. Regarding guide-wire segmentation, $\mathfrak{P}$ is a new approach to the problem that yields significant improvement over state of the art methods. We plan to study in the near future the incorporation of $\mathfrak{P}$ into a complete guide-wire segmentation algorithm.

\section{References}

1. Franken, E., Rongen, P., van Almsick, M., ter Haar Romeny, B.M.: Detection of Electrophysiology Catheters in Noisy Fluoroscopy Images. In: Larsen, R., Nielsen, M., Sporring, J. (eds.) MICCAI 2006. LNCS, vol. 4191, pp. 25-32. Springer, Heidelberg (2006)

2. Bismuth, V., Vancamberg, L., Gorges, S.: A comparison of line enhancement techniques: applications to guide-wire detection and respiratory motion tracking. SPIE Conference Series, vol. 7259 (2009)

3. Barbu, A., Athitsos, V., Georgescu, B., Boehm, S., Durlak, P., Comaniciu, D.: Hierarchical learning of curves application to guidewire localization in fluoroscopy. In: Proc. CVPR, pp. 1-8. IEEE (2007)

4. Honnorat, N., Vaillant, R., Paragios, N.: Guide-Wire Extraction through Perceptual Organization of Local Segments in Fluoroscopic Images. In: Jiang, T., Navab, N., Pluim, J.P.W., Viergever, M.A. (eds.) MICCAI 2010, Part III. LNCS, vol. 6363, pp. 440-448. Springer, Heidelberg (2010)

5. Frangi, A.F., Niessen, W.J., Vincken, K.L., Viergever, M.A.: Multiscale Vessel Enhancement Filtering. In: Wells, W.M., Colchester, A.C.F., Delp, S.L. (eds.) MICCAI 1998. LNCS, vol. 1496, pp. 130-137. Springer, Heidelberg (1998)

6. Meijering, E., Jacob, M., Sarria, J., Steiner, P., Hirling, H., Unser, M.: Design and validation of a tool for neurite tracing and analysis in fluorescence microscopy images. Cytometry Part A 58(2), 167-176 (2004)

7. Vincent, L.: Minimal path algorithms for the robust detection of linear features in gray images. In: Proc. ISMM, pp. 331-338. Kluwer Acad. (1998)

8. Rouchdy, Y., Cohen, L.: Image segmentation by geodesic voting. application to the extraction of tree structures from confocal microscope images. In: ICPR 2008, pp. 1-5. IEEE (2008)

9. Carlotto, M.J.: Enhancement of low-contrast curvilinear features in imagery. IEEE Transactions on Image Processing 16(1), 221-228 (2007)

10. Tankyevych, O.: Filtering of thin objects: applications to vascular image analysis. PhD thesis, Université Paris Est (2010) 